\title{
La incidencia de la accesibilidad al transporte público en el nivel de empleo en la Región Gran Buenos Aires: un modelo de regresión lineal
}

Alejandro Yomal

Maestría en Economía Urbana, Escuela de Gobierno, Universidad Torcuato Di Tella, Argentina

Recibido: 25 de septiembre de 2017. Aceptado: 7 de diciembre de 2017.

\begin{abstract}
Resumen
Aproximadamente, uno de cada cuatro trabajadores residentes de partidos del Gran Buenos Aires (GBA) se desplaza a la Ciudad Autónoma de Buenos Aires (CABA) para trabajar. Estos desplazamientos implican un tiempo de viaje más prolongado y un mayor costo monetario para los bonaerenses, en promedio, que para los residentes de CABA, que trabajan dentro de su propio distrito en un $89 \%$ de los casos. Mediante un modelo de regresión lineal -construido con datos del Censo Nacional de Población, Hogares y Viviendas 2010, datos de la Encuesta de Movilidad Domiciliaria 2009-2010 (ENMODO), y un Índice de Accesibilidad al Transporte Público (IATP) de carácter multidimensional- este estudio pretende inferir que el nivel de accesibilidad al transporte público por parte de los hogares tiene incidencia significativa en los índices de desocupación a nivel radio censal en la Región Gran Buenos Aires (RGBA).
\end{abstract}

The effects of access to public transit on levels of employment in the Greater Buenos Aires Region: a linear regression model
Palabras clave

Transporte Público Empleo

Desempleo

Gran Buenos Aires

Movilidad

\section{Palavras-chave}

Transporte público Emprego

Desemprego

Buenos Aires

Mobilidade

\begin{abstract}
Absract
Approximately one out of four workers residing in Buenos Aires Province commute to Buenos Aires City each day. These commutes imply, on average, a longer and more costly trip for these workers compared to Buenos Aires City workers, who are employed within their district in $89 \%$ of the cases. By means of a linear regression model -built on data from the 2010 Population, Households and Housing National Census (CNPHyV 2010), data from the 2009-2010 Household Commutes Survey (ENMODO), and a multidimensional Public Transit Accessibility Index (IATP) - this study aims to infer that the level of accessibility of households to public transportation has significant incidence on unemployment rates at census tract level in the Greater Buenos Aires Region (RGBA).
\end{abstract}

Keywords

Public Transit Employment Unemployment Buenos Aires Mobility 


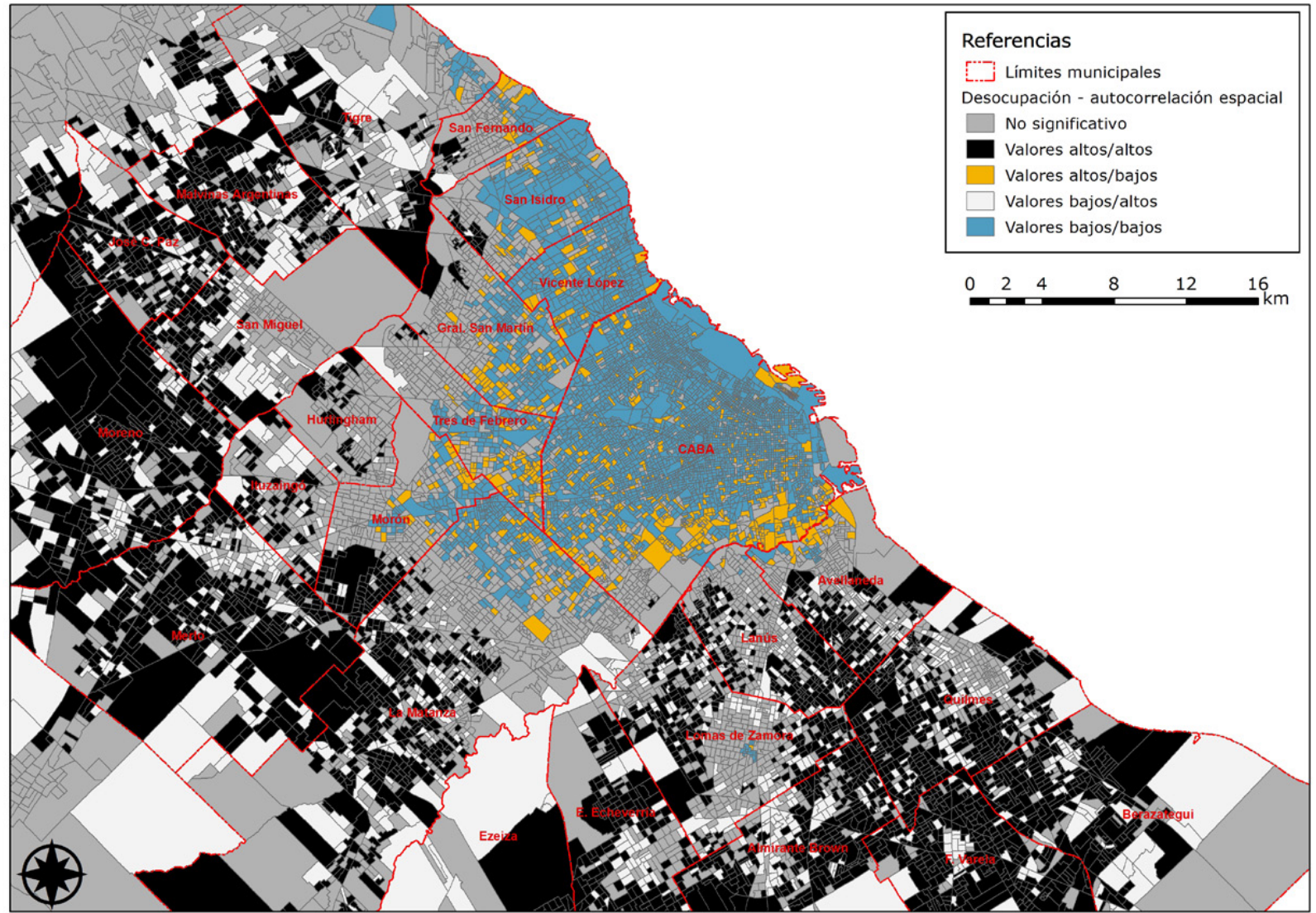

\section{Introducción}

La idea de este trabajo ${ }^{1}$ surgió con el mapa que se muestra a arriba (Figura 1). La distribución de la variable porcentaje de personas desocupadas en el radio censal correspondiente al Censo Nacional de Población, Hogares y Viviendas 2010 (CNPHV 2010) muestra un patrón asociado al territorio de la RGBA.

Se observan radios censales (de aquí en adelante $\mathrm{RC}$ ) con baja prevalencia de desocupación rodeados de $\mathrm{RC}$ con baja desocupación en gran parte del territorio de la $\mathrm{CABA}^{2}$, los partidos de Vicente López, San Isidro y San Fernando, y las primeras coronas de los partidos de La Matanza, Morón, Tres de Febrero, y General San Martín.

Por el contrario, los RC con valores altos de desocupación rodeados de RC con valores altos se encuentran en los sectores más alejados de la CABA, principalmente la segunda y tercera corona de la RGBA: Merlo, Moreno, Malvinas Argentinas, Quilmes, Almirante Brown, Florencio Varela, Berazategui y La Matanza, y sectores de Avellaneda, Lanús, Lomas de Zamora, Esteban Echeverría y Ezeiza. Esta distribución nos estaría indicando a priori que la desocupación tiene un comportamiento asociado al territorio, que guarda relación con diversos factores, entre los que se incluyen la accesibilidad al transporte público.

La hipótesis central de este trabajo sostiene que el grado de accesibilidad al transporte público por parte de los hogares influye significativamente en los niveles de empleo de éstos. Lo que subyace en esta hipótesis es que el desempleo no es solo resultado de las capacidades técnicas de los individuos y de procesos económicos estructurales, sino que existen factores espaciales (localización) y temporales (tiempo de viaje al trabajo) que pueden facilitar o restringir el acceso al mercado de trabajo por parte de los demandantes de empleo.
Figura 1. Desocupación en la RGBA - Autocorrelación Espacia Local Anselin Moran's. Fuente: Elaboración propia en base a datos del CNPHV 2010 y el Instituto Geográfico Nacional (IGN).

1. Artículo adaptado de la tesis de Maestría en Economía Urbana, Universidad Torcuato Di Tella.

2. Exceptuando sectores de la zona sur y la Villa 31/31bis. 
En base a esto, proponemos las siguientes líneas de investigación:

¿Cómo incide la infraestructura de transporte público en los niveles de empleo en la Región Gran Buenos Aires ${ }^{3}$ (RGBA)?

" ¿Cómo se puede medir la relación entre las tasas de desocupación a nivel radio censal y el nivel de accesibilidad de los hogares a la red de infraestructura de transporte público?

»Cuáles son los determinantes sociodemográficos de la desocupación en la RGBA?

Una parte importante de este estudio constituye un ejercicio metodológico que consiste en procesar y combinar bases de datos de dos relevamientos (CNPHV 2010, ENMODO 2010) para incorporar datos de accesibilidad y uso del transporte público a la unidad geográfica mínima de análisis, el radio censal. En este sentido, un objetivo central es diseñar un Índice de Accesibilidad al Transporte Público (IATP) que permita conocer el nivel de accesibilidad al transporte público de los hogares de cada uno de los radios censales de la RGBA.

El IATP, junto al tiempo promedio de viaje al trabajo y tres variables sociodemográficas censales - Necesidades Básicas Insatisfechas $(\mathrm{NBI})^{4}$, porcentaje de personas que no asisten o asistieron a la escuela secundaria y porcentaje de hogares con tenencia formal de la vivienda - se incorporarán a un modelo de regresión lineal que permitirá cuantificar la incidencia de cada una de las variables en las tasas de desocupación a nivel radio censal. Aunque existen otras variables que puedan incidir sobre las tasas de desocupación, se priorizó trabajar con datos desagregados a nivel radio censal y de fuentes oficiales y confiables. De todas formas, el indicador NBI incluido en el modelo abarca múltiples dimensiones que pueden estar influyendo sobre el nivel de desocupación.

La regresión lineal no solo permite trabajar con gran cantidad de casos de manera sencilla (12.685 en este estudio), sino también calcular el efecto de cada una de las variables sobre aquella que se quiere explicar: el nivel de desocupación. La técnica de la regresión es útil para esbozar un modelo general y encontrar tendencias con un efecto cuantificable; por ello resulta la herramienta indicada para los fines de este estudio, que propone una metodología concreta para medir la asociación entre accesibilidad al transporte público y las tasas de desocupación a nivel radio censal, replicable en aglomerados urbanos de otras regiones con características similares a la RGBA.

\section{Antecedentes y marco teórico}

\section{La formación de la RGBA y el sistema de transporte público}

El comienzo de la formación de la Región Gran Buenos Aires puede ubicarse entre 1870 y 1930, cuando se estructura una conformación monocéntrica y radial - con eje en la zona del puerto de la ciudad de Buenos Aires (CABA) - que será la base del crecimiento que continúa hasta hoy. En esta etapa, en la cual se delimitan las características de producción, movilidad y crecimiento de la urbe, la región experimenta un acelerado crecimiento poblacional debido a la ola de inmigración europea. El área central se expande y consolida, y se producen asentamientos poblacionales alrededor de las estaciones de los distintos ramales del ferrocarril, que se convierte en el primer estructurador metropolitano (CAF, 2011:21).

Entre 1930 y 1970 continúa el crecimiento poblacional (en 1947 la población es el doble con respecto a 1914) e industrial, y se consolidan los cordones urbanos que rodean
3. La RGBA definida por el INDEC (Instituto Nacional de Estadística y Censos) incluye la Ciudad Autónoma de Buenos Aires (CABA) y 24 partidos del conurbano bonaerense: Almirante Brown, Avellaneda, Berazategui, Esteban Echeverría, Ezeiza, Florencio Varela, General San Martín, Hurlingham, Ituzaingó, José C. Paz, La Matanza, Lanús, Lomas de Zamora, Malvinas Argentinas, Merlo, Moreno, Morón, Quilmes, San Fernando, San Isidro, San Miguel, Tigre, Tres de Febrero y Vicente López.

4. El INDEC considera que un hogar tiene Necesidades Básicas Insatisfechas cuando se cumplen al menos una de las siguientes condiciones: que el hogar resida en habitaciones de inquilinato, hotel o pensión, en viviendas no destinadas a fines habitacionales o viviendas precarias; que el hogar no posea retrete; que exista hacinamiento crítico (3 personas o más por cuarto); que haya al menos un niño en edad escolar que no asista a la escuela; o que haya 40 más personas por miembro ocupado y el jefe de hogar tenga bajo nivel de educación (dos años o menos en el nivel primario). 
a la CABA, conocidos como el conurbano. Desde 1947 la población de la ciudad se estabiliza en unos tres millones de habitantes (hoy 2.890.151), que representan un $19 \%$ de la población de todo el país, mientras que en los partidos que en ese momento formaban el conurbano se concentraban 1,8 millones de personas ( $10 \%$ de la población argentina). A partir de este momento el crecimiento demográfico neto se produce en la RGBA, impulsado por procesos migratorios campo-ciudad e interior-capital que resultan en la consolidación de la primera corona metropolitana y en la densificación de las áreas centrales. En 1960 los partidos del conurbano habían duplicado su población con respecto a 13 años atrás, alcanzando 4,3 millones de personas, equivalente al $20 \%$ del total nacional. Para el año 1970 esta población alcanzaba 5,5 millones de personas, mientras que la población de la CABA se mantenía inalterada (CAF, 2011:22).

En 1980 la población total de la RGBA asciende a 10,8 millones de habitantes y el conglomerado alcanza una tercera corona de expansión territorial. Además, se acentúan las tendencias de segregación socioterritorial que continúan en la actualidad, con el crecimiento de clases medias y alta en la zona norte, impulsado por las autopistas, y el crecimiento de sectores populares hacia el sur, de la mano de los ferrocarriles (CAF, 2011:23).

Actualmente la población de la RGBA alcanza los 12.806.866 habitantes (CNPHV 2010), siendo la segunda área metropolitana más poblada de América del Sur después de San Pablo, Brasil. Su sistema de transporte público es diverso, y a excepción del subterráneo, tiene alcance en gran parte de la región.

El transporte urbano en la RGBA está compuesto por servicios de colectivos (autobús) de jurisdicción nacional, provincial y municipal que conforman 313 rutas, seis líneas de subterráneo con una línea de premetro (tranvía) en el sur de la CABA y siete líneas de ferrocarril (CAF, 2011:27).

El 43\% del total de los viajes realizados en la RGBA son en transporte público, el 26\% en transporte privado y el 31\% es no motorizado (ENMODO, 2012:26). La proporción de viajes realizados en transporte público es relativamente alta si comparamos con otras ciudades de América Latina ${ }^{5}$ (CAF, 2011): en Bogotá es del 57\% (2011:146), en Lima es del 53\% (2011:227), en Belo Horizonte alcanza el 44\% (2011:40), en Porto Alegre es del 42\% (2011:81), en Santiago de Chile 36\% (2011:132) en San Pablo 33\% (2011:117), en Guadalajara alcanza el 31\% (2011:196), mientras que en Curitiba los viajes en transporte público representan el 28\% del total (2011:62).

El subsistema de colectivos o autobuses es el que más pasajeros mueve en la RGBA. En 2015, sus 9.892 unidades distribuidas en 138 líneas (en sus múltiples ramales) transportaron 1.621 millones de pasajeros pagos, equivalente a 4,4 millones por día en promedio (DGEyC, 2017:543). El 71\% de los viajes realizados en modo público en la RGBA se hacen en este medio de transporte (CNRT, 2017:24). Recientemente se han construido carriles exclusivos y estaciones en ocho arterias de la CABA y una del partido de La Matanza para agilizar el tránsito de colectivos, en lo que se ha dado en llamar Metrobus.

El subsistema de ferrocarril alcanza los $828 \mathrm{~km}$ de tendido (el 20\% está electrificado) y ostenta 250 estaciones y 4 terminales en la CABA (Retiro, Constitución, Once y Chacarita) (CAF, 2011:27). En 2016, la red de superficie de las siete líneas del ferrocarril transportó un total de 357,9 millones de pasajeros pagos, un 8,6\% más que al año anterior (CNRT, 2017:13). Esto equivale a 977.868 pasajeros diarios en promedio. El $16 \%$ de los viajes realizados en modo público en la RGBA se hacen en alguno de los siete ramales de ferrocarril metropolitano (CNRT, 2017:24).

El subsistema de subterráneo y premetro, que opera solamente en la CABA, posee $87,3 \mathrm{~km}$ de red y 127 estaciones. El 13\% de todos los viajes realizados en modo público en la RGBA 
se hacen en subterráneo (CNRT, 2017:24). En 2016 transportó unos 304 millones de pasajeros pagos, que representa un aumento del $11 \%$ con respecto al año anterior (Metrovías, 2017:3). El promedio diario de pasajeros pagos transportados equivale a 832.723, aunque sumando los pasajeros que no pagan esta cifra se eleva a 1.201.053 (DGEyC, 2017:540). Pese a que el tendido de subterráneo $(87,3 \mathrm{~km})$ representa el $10,5 \%$ del tendido del ferrocarril $(828 \mathrm{~km})$, la cantidad de pasajeros trasportados por subterráneo es algo mayor que los transportados por ferrocarril (977.868 pasajeros diarios); esto evidencia la importancia de la red de subterráneo para la movilidad en el área central de la RGBA.

\section{Accesibilidad al sistema de transporte público}

El estudio de la accesibilidad a servicios públicos de transporte por parte de la población se ha incrementado en los últimos años. Lucas et al. (2016) proponen una revisión bibliográfica con el objetivo de identificar los distintos conceptos y definiciones que se utilizan para trabajar el problema. Dos de esos conceptos son útiles para enmarcar la problemática que aborda este estudio: pobreza de movilidad y pobreza de accesibilidad ${ }^{6}$. El concepto de pobreza de movilidad alude a "una carencia sistémica de transporte (generalmente motorizado) que genera dificultades en la movilidad, a menudo (pero no siempre) ligado a una carencia de servicios o infraestructura" (Lucas, 2016:355) ${ }^{7}$. El concepto de pobreza de accesibilidad alude a "la dificultad de alcanzar ciertas actividades claves - tales como el empleo, la educación, los servicios de salud, locales comerciales, entre otros - en tiempo, forma y costo razonables" (Lucas, 2016:355) ${ }^{8}$. La accesibilidad a la red de transporte público en un aglomerado como la RGBA resulta fundamental en el acceso por parte de la población tanto a los bienes y servicios urbanos (salud, educación, cultura, esparcimiento, etc.) como al mercado de empleo. Como describen Andrea Gutiérrez y Julio Rearte (2006:830) siguiendo a R. Bertoncello,

\section{[...] la movilidad territorial de los distintos grupos sociales se manifiesta} periódicamente en las áreas urbanas. Las migraciones diarias demandan servicios de transporte colectivos y medios particulares que concurren en determinados horarios y lugares y se disputan el espacio urbano. La localización diferenciada de los lugares de residencia, trabajo y estudio es, en términos generales, su principal causa. Esta movilidad territorial de la población es uno de los fenómenos que mejor describe a las sociedades modernas. En la Región Metropolitana de Buenos Aires, esta movilidad presenta una dinámica pendular y una configuración en radios que vinculan el área central con el resto de la región. Esta estructura radio céntrica de los desplazamientos está vinculada al estilo de desarrollo socio-económico iniciado a partir del período independiente de la nación, y perdura hasta hoy. Es así que las redes de servicios públicos de transporte masivo por automotor, ferrocarril y subte continúan atendiendo el patrón básico de centro-periferia estructurado por la ciudad industrial. La CABA, por su parte, sigue albergando el núcleo central de generación y atracción de viajes de la región metropolitana.

La falta de accesibilidad al transporte puede acarrear serias consecuencias en la situación socioeconómica de los hogares que residen a grandes distancias de los centros de empleo, donde el valor del suelo es más accesible. Según Smolka y Biderman (2011:10),

Al mudarse a áreas más distantes (con precios menores del suelo, pero mayores gastos de transporte), las familias de bajos ingresos evitan los costos iniciales más altos de vivir en áreas intermedias más consolidadas. Las estrategias de crédito actuales de las familias de bajos ingresos por lo tanto involucran un trueque de gastos de transporte por tamaño de predio y procesos de construcción paulatina.

Para aquellos hogares que se asientan en zonas alejadas de los centros de empleo, los gastos en transporte y tiempos de viaje pueden operar como impedimentos al acceso a
6. Traducido del inglés: mobility poverty, accessibility poverty.

7. Traducción propia.

8. Traducción propia. 
mejores oportunidades laborales. Un demandante de empleo puede optar por changas o trabajos temporarios, generalmente informales, en su lugar de residencia o en zonas aledañas, que no le otorgan la seguridad y previsibilidad que brindan en mayor medida los trabajos formales en los centros de empleo.

A su vez, hay una serie de determinantes sociodemográficos que también funcionan como impedimentos para acceder a oportunidades laborales. Siguiendo a Jorge Blanco (2016:99),

[...] la movilidad puede ser analizada ventajosamente en el marco de las características y estrategias de los grupos familiares. Los niveles de ingresos, la escolaridad, la estructura etaria del hogar, el momento en el ciclo de vida, las relaciones de género, son factores que condicionan las decisiones de movilidad (también las de localización) y que brindan un marco más adecuado para el análisis que la consideración del individuo aislado.

El indicador de Necesidades Básicas Insatisfechas, que mide multidimensionalmente las condiciones de pobreza estructural de los hogares, el porcentaje de personas que no asisten o asistieron a la escuela secundaria y la tenencia formal de la vivienda, entre otros, son factores que en mayor o menor medida pueden incidir en las tasas de desocupación de los hogares. Existe evidencia empírica que asocia un menor nivel educativo con mayores tasas de desocupación. En el cuarto trimestre de 2016, la desocupación promedio en CABA alcanzaba el 8\%; más aún, era del 12\% en personas con secundario incompleto o menos, $8,2 \%$ en personas con secundario completo y $4 \%$ en personas con nivel superior completo ${ }^{9}$ (DGEyC, 2017b:2). A su vez, en partidos del Gran Buenos Aires, la tasa de desocupación en el segundo trimestre de 2015 era de 7,9\%: 10,6\% en personas con secundaria incompleta, 9,3\% en personas con secundaria completa y $3,3 \%$ en personas con nivel superior completo ${ }^{10}$.

Stanley et al. (2011:791) exploran los vínculos entre movilidad y exclusión social, conceptualizada a través de cinco dimensiones: ingresos del hogar, condición de actividad (empleado, desempleado, cursando estudios), participación política (partidos políticos, agrupaciones barriales, organizaciones de la sociedad civil), participación cultural (uso de bibliotecas, asistencia y/o participación en eventos culturales y deportivos, grupos de interés, etc.) y sostén social (posibilidad de obtener ayuda de familiares, amigos o vecinos). Tomando datos de encuestas de movilidad realizadas en Melbourne, Australia, los autores encontraron una asociación significativa entre una mayor movilidad (viajes realizados/actividades realizadas) y un menor riesgo de exclusión social, tanto a nivel metropolitano como regional. Además, encontraron que mejorar la movilidad en sí misma puede ser una manera de estimular el desarrollo de capital social, lo que le otorga a la realización de viajes un potencial rol directo e indirecto de reducir el riesgo de exclusión social (Stanley et al., 2011:799).

Con el objetivo de medir la distribución espacial de la necesidad de transporte público e identificar brechas en la calidad de la provisión de transporte público en Hobart, Australia, Currie (2004) propone un abordaje en el cual cuantifica la distribución de necesidades de una comunidad con un índice de necesidades de transporte. Este índice lo sintetiza ponderando una serie de indicadores que obtiene de datos censales: adultos sin automóvil, personas mayores de 60 años, personas con pensión por discapacidad, adultos con bajos ingresos, adultos inactivos y estudiantes (Currie, 2004:141). El autor diseña un modelo de red de transporte público que mide la accesibilidad que tiene la población al sistema de autobuses y utiliza técnicas de información geográfica para mostrar la distribución de las brechas identificadas entre servicios y necesidades. El modelo considera la calidad del viaje en transporte público (rutas, horas de ingreso y egreso, frecuencias, tiempos de viaje y tarifas) para distintos momentos de la semana
9. Terciario, superior no universitario y universitario.

10. Elaboración propia con bases de microdatos de la Encuesta Permanente de Hogares (EPH). 
a catorce lugares clave (área central, comercios, universidades, farmacias, escuelas, hospitales, entre otros) (Currie, 2004:139). Como resultado, el modelo genera datos para mapear las zonas de la ciudad según los distintos grados de necesidad de transporte público de la comunidad y la provisión de transporte público, identificando los sectores más perjudicados (necesidad alta de transporte público y provisión baja de transporte público) y más favorecidos. Lo interesante de este abordaje es que el autor complementa el diagnóstico territorial de provisión de transporte público con un índice de necesidades de transporte público de acuerdo a datos censales, con el fin de medir la brecha entre la necesidad y el servicio.

En un intento por comprender la asociación entre la disponibilidad de transporte público y el acceso a oportunidades de empleo en la región metropolitana de Chicago, Minocha et al. (2008) diseñan un modelo con dos indicadores: transit availability index (TAI) o índice de disponibilidad del transporte y regional transit employment accessibility index (TEAI) o índice regional de acceso a empleos por transporte.

La oferta de transporte público se sintetiza en el TAI, que combina el número de residentes a distancia caminable de paradas de colectivo o estaciones de subte o tren con información sobre la frecuencia, horas de servicio y cobertura geográfica. Estas medidas representan el nivel de accesibilidad en determinada parada o estación del sistema de transporte público. El TEAI representa el potencial que tienen los residentes de cada zona de análisis de transporte (traffic analysis zone - TAZ) para acceder a empleos localizados en otras TAZ de la región.

Los autores encuentran que hay una gran variabilidad en la disponibilidad y uso del transporte público en la región metropolitana de Chicago, con la consiguiente variación en el nivel de accesibilidad y uso del sistema de transporte y su eficacia para permitir el acceso de los residentes a los centros de empleo. La comparación de ambos índices permite a los autores identificar zonas en las cuales hay disparidad entre la disponibilidad y la demanda de transporte público para acceder a zonas de empleo utilizando cuatro categorías de análisis: 1) nivel alto de disponibilidad de transporte y nivel alto de acceso a empleos por transporte; 2) nivel alto de disponibilidad de transporte y nivel bajo de acceso a empleos por transporte; 3 ) nivel bajo de disponibilidad de transporte y nivel alto de acceso a empleos por transporte; y 4) nivel bajo de disponibilidad de transporte y nivel bajo de acceso a empleos por transporte.

El presente estudio se inspira, a su vez, en el trabajo de Gabriel y Rosenthal (1996), que relaciona los tiempos de viaje al trabajo de los distintos grupos raciales en Estados Unidos y el acceso a oportunidades de empleo. Supone que una mayor distancia (y tiempo de viaje) a los centros de empleo debería implicar una compensación monetaria reflejada en menores precios de los inmuebles y en diferentes niveles de amenidades para aquellos hogares cuyos trabajadores deben viajar más tiempo a sus empleos. Descubren que, controlando por efectos de barrio e ingresos y a un mismo nivel de instrucción, los trabajadores de raza negra tienen tiempos de viaje significativamente mayores que los blancos y asiáticos, debido a restricciones de acceso a ciertos lugares de residencia y empleo. A su vez, descubren que la movilidad de los hogares es un mecanismo importante a través del cual el mercado alcanza equilibrio espacial, lo que aportaría evidencia en favor de los argumentos que indican que la proximidad restringida a oportunidades residenciales y de empleo reduce el bienestar económico de los hogares minoritarios.

Algo similar podría darse en la RGBA, donde los hogares de menores recursos se asientan en mayor medida en el suelo más accesible de las zonas periféricas a los centros de empleo, aunque a cambio deben solventar el costo monetario y temporal de trasladarse diariamente a sus empleos. 
Según datos de la Dirección General de Estadística y Censos (DGEyC, 2013), en la RGBA hay aproximadamente seis millones de empleos, de los cuales 3.700 .000 $(61,7 \%)$ están en los 24 partidos del conurbano bonaerense (GBA) y 2.300 .000 $(38,3 \%)$ en CABA.

De los 2.300.000 empleos de CABA, el 47,7\% (1.205.000) son ocupados por residentes de GBA. Las 1.205.000 personas que deben trasladarse a CABA para trabajar representan el 27\% del total de los 4.450 .000 trabajadores residentes de GBA. Por otro lado, de los trabajadores que residen en CABA solamente el 11\% trabajan en GBA, tal como figura en el Cuadro 2.

Cuadro 1. Distribución de trabajadores de la RGBA según lugar de trabajo. Nota: *285 mil personas trabajan en ambos lugares. Fuente: Elaboración propia en base a DGEyC (2013).

\begin{tabular}{|l|c|c|c|}
\hline Región & $\begin{array}{c}\text { Total de } \\
\text { empleos }\end{array}$ & $\begin{array}{c}\text { \% de trabajadores } \\
\text { residentes de GBA }\end{array}$ & $\begin{array}{c}\text { \% de trabajadores } \\
\text { residentes de CABA }\end{array}$ \\
\hline CABA & $2.525 .000^{*}$ & $47,7 \%$ & $52,3 \%$ \\
\hline Partidos GBA & 3.700 .000 & $93,8 \%$ & $6,2 \%$ \\
\hline Total RGBA & 6.000 .000 & $78 \%$ & $22 \%$ \\
\hline
\end{tabular}

Cuadro 2. Distribución de trabajadores de la RGBA según lugar de residencia. Fuente: Elaboración propia en base a DGEyC (2013).

\begin{tabular}{|l|c|c|c|}
\hline Lugar de residencia & Total de trabajadores & $\begin{array}{c}\text { \% que trabaja en } \\
\text { CABA }\end{array}$ & \% que trabaja en GBA \\
\hline CABA & 1.500 .000 & $89 \%$ & $11 \%$ \\
\hline Partidos GBA & 4.450 .000 & $27 \%$ & $73 \%$ \\
\hline Total RGBA & 5.950 .000 & $38,3 \%$ & $61,7 \%$ \\
\hline
\end{tabular}

A su vez, los residentes de CABA que trabajan en GBA tienen mejores condiciones laborales que los residentes de GBA que trabajan en CABA:

Los residentes en Partidos del GBA que trabajan en la CABA tienen un salario por hora $21 \%$ más bajo que el de sus pares porteños y trabajan en promedio 1 hora más por semana. Los porteños que trabajan afuera de su distrito, en cambio, tienen un ingreso horario $21 \%$ superior $y$ trabajan 7 horas menos por semana que sus pares que viven en Partidos del GBA. (DGEyC, 2013:13).

Siguiendo a Gabriel y Rosenthal (1996), estos datos nos estarían indicando que, en términos muy generales, el tiempo y costo de viaje adicionales que deben solventar los residentes de CABA que trabajan en GBA son compensados en mayor medida (ganan $21 \%$ más y trabajan 7 horas semanales menos que sus pares bonaerenses) que el tiempo y costo de viaje adicionales que experimentan los residentes de GBA que trabajan en CABA (ganan 21\% menos y trabajan 1 hora semanal más que sus pares porteños).

Si consideramos lo visto hasta el momento, queda en evidencia la concentración del mercado laboral en la ciudad, que nuclea el 38,3\% de los empleos de la región, con uno de cada cuatro (27\%) trabajadores bonaerenses desplazándose hacia la CABA para trabajar. La red de infraestructura de transporte público, como hemos visto, juega un papel preponderante en estos desplazamientos y, según la hipótesis de este estudio, el acceso a ella impacta de manera significativa en el acceso a oportunidades de empleo $y$, por ende, en el nivel de desempleo. 


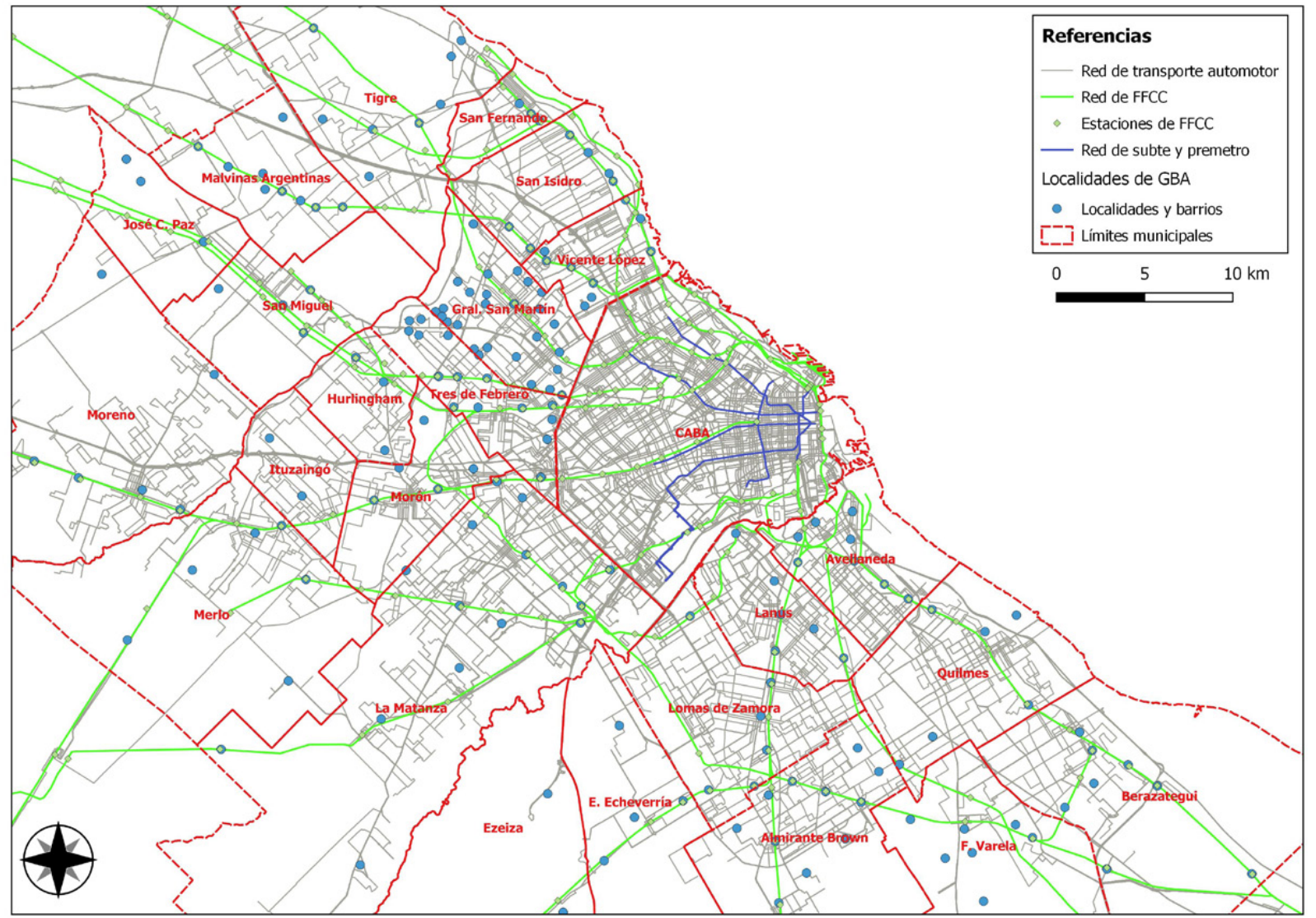

\section{Metodología}

Para explorar los factores que inciden en los niveles de desempleo - nuestra variable dependiente - en el territorio de la RGBA, se diseñó un modelo de regresión lineal utilizando la base georreferenciada de 12.685 radios censales con tres variables provenientes del CNPHV 2010 que funcionan como variables independientes: Necesidades Básicas Insatisfechas, porcentaje de personas que no asisten o asistieron a la escuela secundaria y porcentaje de hogares con tenencia formal de la vivienda. Se incorporó además a dicha base el Índice de Accesibilidad al Transporte Público y el tiempo de viaje al trabajo promedio, ambas variables construidas para cada uno de los radios censales a partir de las bases de datos de la ENMODO 2010.

Como insumo básico para el diseño del IATP se investigó qué datos relacionados a la red de transporte de la región se encontraban georreferenciados. Se tomaron las capas de estaciones de subterráneo, Metrobus y ferrocarril, y de recorridos de colectivos de la página de Buenos Aires Data del Gobierno de la Ciudad de Buenos Aires http://data.buenosaires.gob.ar. Se encontró que la capa de estaciones de ferrocarril estaba incompleta, complementándose con datos del Instituto Geográfico Nacional (IGN). A su vez, se encontró que los datos geográficos de recorridos de colectivos solamente incluían líneas que tenían al menos parte del recorrido en la CABA. Por ende, para complementar esta información, se añadió la capa de recorridos de colectivos de Open Street Map (http://mapzen.com) y se procedió a completar las líneas faltantes con un relevamiento manual y minucioso, utilizando como guía de recorridos la página http://omnilineas.com.ar. Esta información georreferenciada sirvió como insumo para hacer los cálculos requeridos para la construcción del IATP.
Figura 2. Red de transporte público, localidades y barrios de la RGBA. Fuente: Elaboración propia en base a datos de ENMODO 2010 CNPHV 2010, IGN, Open Street Map, Omnilíneas y de relevamiento propio. 
Se diseñó el Índice de Accesibilidad al Transporte Público con la idea de abarcar, mediante cuatro variables, desagregado por radio censal, el nivel de accesibilidad a las redes de transporte público que presentan los hogares de acuerdo a su ubicación en la región. La estructura del IATP se muestra en el Cuadro 3:

Cuadro 3. Composición del IATP. Fuente: Elaboración propia.

\begin{tabular}{|l|l|c|}
\hline Variable & Descripción & Ponderación \\
\hline dens_cole & $\begin{array}{l}\text { Suma de la longitud (en metros) de los recorridos de los } \\
\text { colectivos/Metrobus del RC / Densidad demográfica del RC } \\
\text { (personas/hectárea) }\end{array}$ & 0,2 \\
\hline dist_cole & $\begin{array}{l}\text { Distancia (en metros) del centroide del RC al recorrido de } \\
\text { colectivo/Metrobus más cercano }\end{array}$ & 0,3 \\
\hline dist_tren & $\begin{array}{l}\text { Distancia (en metros) desde el perímetro del RC a la estación de } \\
\text { ferrocarril más cercana }\end{array}$ & 0,3 \\
\hline dist_subte & $\begin{array}{l}\text { Distancia (en metros) desde el perímetro del RC a la estación de } \\
\text { subte o premetro cercana }\end{array}$ & 0,2 \\
\hline
\end{tabular}

Los valores correspondientes a las cuatro variables se segmentaron por decil (del $1 \mathrm{al}$ 10), se multiplicaron por el factor de ponderación y se sumaron para dar como resultado el IATP, cuyo valor 1 indica el menor nivel de accesibilidad al transporte público, y el valor 10 el mayor acceso a éste.

La variable dens_cole se calculó sumando la longitud de los recorridos de los colectivos en cada RC dividiendo por la densidad demográfica correspondiente, de tal manera de ponderar el valor por la población y por el área de cada RC. Las tres variables de distancia (dist_cole, dist_tren, dist_subte) se calcularon utilizando la función near del ARCMap, que asigna a la tabla de atributos de los radios censales de la RGBA la distancia en metros a la cual se encuentra cada uno de los radios censales a los tres objetos geográficos en análisis: 1) recorridos de colectivos, 2) estaciones de ferrocarril y 3) estaciones de subterráneo y premetro.

Como complemento al IATP para la dimensión de movilidad se buscó incorporar al modelo el tiempo de viaje promedio al trabajo de los miembros de los hogares. Para esto, la fuente más actual y desagregada resultó ser la Encuesta de Movilidad Origen Destino 2009/2010 (ENMODO), llevada adelante por la Secretaría de Transporte de la Nación, que relevó mediante una muestra probabilística los viajes diarios de los habitantes de la RGBA ampliada. Tomando solamente los viajes con destino lugar de trabajo o asunto laboral se calculó, para los habitantes de la RGBA, un tiempo de viaje promedio de 45,8 \pm 41,6 minutos. En el Cuadro 3 se muestran los promedios de tiempos de viaje al trabajo por partido. 
Cuadro 3. Promedios de tiempo de viaje al trabajo y cantidad de viajes según partido de residencia. Fuente: Elaboración propia en base a datos de ENMODO.

\begin{tabular}{|c|c|c|c|}
\hline Partido & Viajes al trabajo & Promedio (min) & Desvío estándar \\
\hline San Isidro & 102.321 & 37,04 & 25,12 \\
\hline CABA & 1.106 .443 & 37,3 & 23 \\
\hline General San Martin & 145.818 & 38,56 & 27,8 \\
\hline Vicente López & 116.838 & 40,03 & 50,41 \\
\hline Avellaneda & 107.651 & 42,59 & 27,75 \\
\hline San Fernando & 55.561 & 43,05 & 41,84 \\
\hline Tres de Febrero & 111.273 & 43,21 & 68,68 \\
\hline Lanús & 160.184 & 43,45 & 41,34 \\
\hline Tigre & 90.772 & 45,27 & 56,14 \\
\hline Morón & 103.193 & 45,34 & 32,49 \\
\hline Lomas de Zamora & 181.177 & 47,49 & 39,18 \\
\hline Quilmes & 148.956 & 48,1 & 35,65 \\
\hline Esteban Echeverría & 68.720 & 48,14 & 73,01 \\
\hline Ituzaingó & 53.960 & 48,37 & 36,66 \\
\hline Almirante Brown & 147.552 & 48,7 & 39,93 \\
\hline Hurlingham & 49.100 & 49,48 & 33,14 \\
\hline Berazategui & 88.115 & 50,63 & 38,37 \\
\hline Ezeiza & 32.442 & 53,24 & 49,04 \\
\hline San Miguel & 74.560 & 54,06 & 37,91 \\
\hline La Matanza & 397.685 & 54,69 & 44,05 \\
\hline Malvinas Argentinas & 88.781 & 56,23 & 71,63 \\
\hline José C. Paz & 58.190 & 57,56 & 51,96 \\
\hline Florencio Varela & 78.331 & 60,16 & 41,67 \\
\hline Merlo & 122.811 & 64,09 & 45,69 \\
\hline Moreno & 99.863 & 66,24 & 75,27 \\
\hline 24 partidos GBA & 2.638 .854 & 49,4 & 45,2 \\
\hline Total RGBA & 3.790 .298 & 45,83 & 41,57 \\
\hline
\end{tabular}

Aunque los resultados se muestran a nivel partido, el procesamiento se hizo a nivel barrio/localidad según el relevamiento de ENMODO, abarcando 200 localidades y barrios de la RGBA (incluidos los 48 barrios de la CABA) y calculándose el promedio de duración en minutos del total de viajes realizados desde cada una de las unidades geográficas hacia los lugares de empleo. Se utilizó la capa de centros urbanos del IGN y se georreferenciaron, con el auxilio de imágenes satelitales, las localidades y los barrios faltantes, según aquellos relevados en la ENMODO. Ante la imposibilidad de contar con los límites geográficos precisos de cada uno de los barrios, localidades y ciudades al interior de cada partido, se optó por estimarlos utilizando el procedimiento de polígonos de Thiessen ${ }^{11}$, con el fin de poder asignar el tiempo de viaje al trabajo promedio de cada localidad o barrio a sus radios censales correspondientes mediante un join (unión) espacial. Este procedimiento permitió incorporar al modelo de regresión el tiempo de viaje al trabajo promedio como variable independiente.
11. Los polígonos de Thiessen se basan en la distancia euclidiana y se generan al unir los puntos entre sí, trazando las mediatrices de los segmentos de unión. Las intersecciones de estas mediatrices determinan una serie de polígonos en un espacio bidimensional alrededor de un conjunto de puntos de control, de manera que el perímetro de los polígonos generados sea equidistante a los puntos vecinos y designan su área de influencia. 


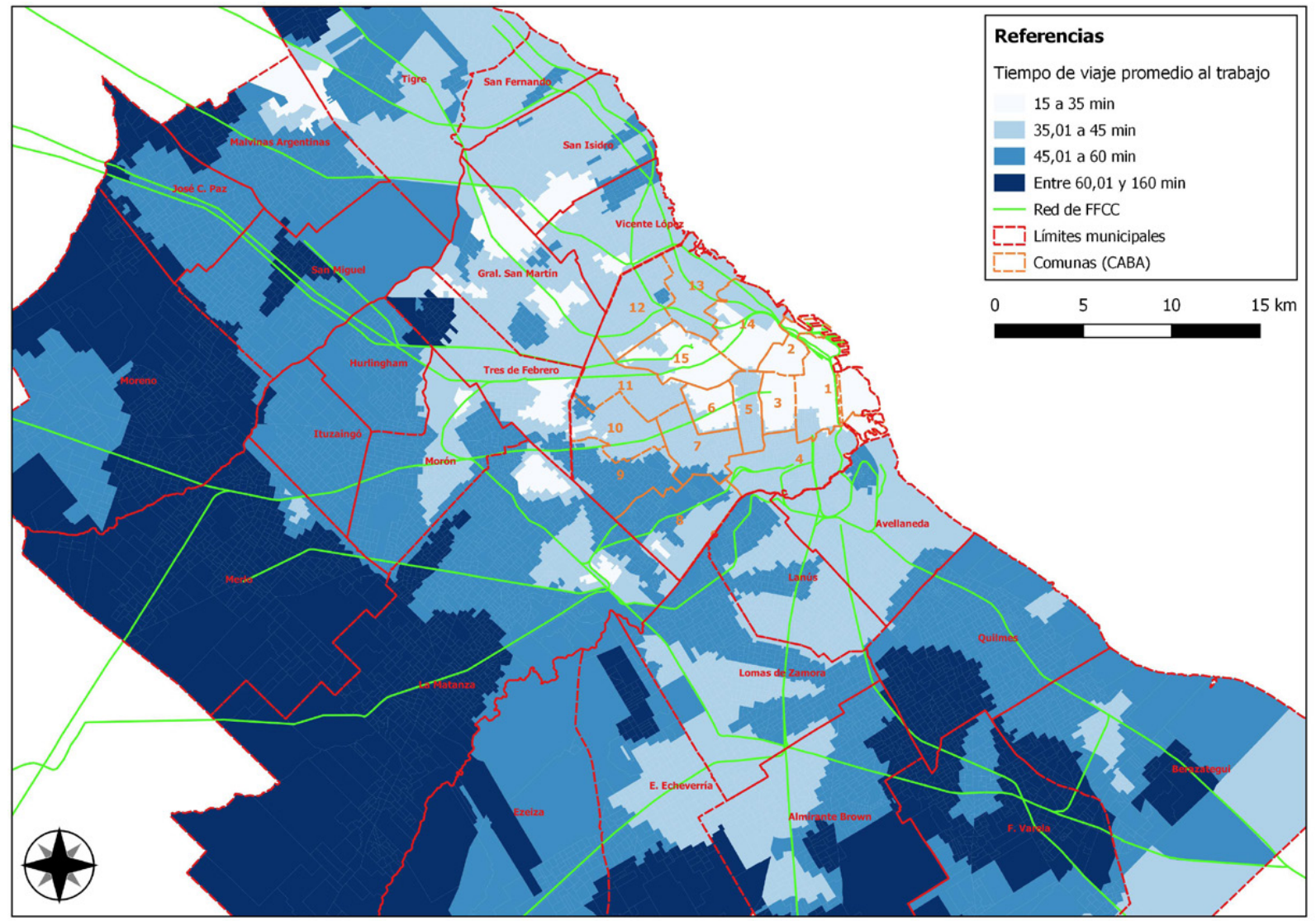

\section{Análisis}

\section{Tiempos de viaje diferenciales}

La Figura 3 ilustra los resultados del relevamiento del procesamiento de las bases de la ENMODO que arrojó el tiempo promedio de viaje por barrio/localidad/ciudad, en este caso estimado para todo el territorio de la RGBA.

La tendencia general es un aumento del promedio de tiempo viaje a medida que nos alejamos del centro de la Ciudad de Buenos Aires, el sitio de la RGBA con mayor concentración de empleos.

Específicamente, en la Ciudad de Buenos Aires los mayores tiempos de viaje se observan en los trayectos originados en el barrio de Coghlan (noreste de la Comuna 12) y en las comunas 8 y 9 con una duración promedio de 45 minutos a 1 hora, siendo la excepción Villa Riachuelo (sur de la Comuna 8), que ostenta un promedio de 29 minutos; esto último tal vez sea un indicador de que una menor proporción de los habitantes del barrio se desplacen al centro de la ciudad para trabajar. En las comunas 4, 5, 7, 10, 11, 12 y 13 el tiempo de viaje oscila entre los 35 y 45 minutos en promedio. Por último, en gran parte del territorio de las comunas 1, 2, 3, 6, 14 y 15 el tiempo de viaje promedio no supera los 35 minutos.

San Isidro es el único partido que tiene un tiempo de viaje al trabajo promedio menor que CABA: 37 minutos. En los partidos de General San Martin, Vicente López, Avellaneda, San Fernando, Tres de Febrero y Lanús el tiempo de viaje promedio oscila entre los 38 y 44 minutos. En los partidos de Tigre, Morón, Lomas de Zamora, Quilmes, Esteban Echeverría, Ituzaingó, Almirante Brown, Hurlingham,
Figura 3. Promedio de tiempo de viaje al trabajo (minutos) en la RGBA, y red ferroviaria. Fuente: Elaboración propia en base a datos de ENMODO 2010, CNPHV 2010, Buenos Aires Data e IGN. 


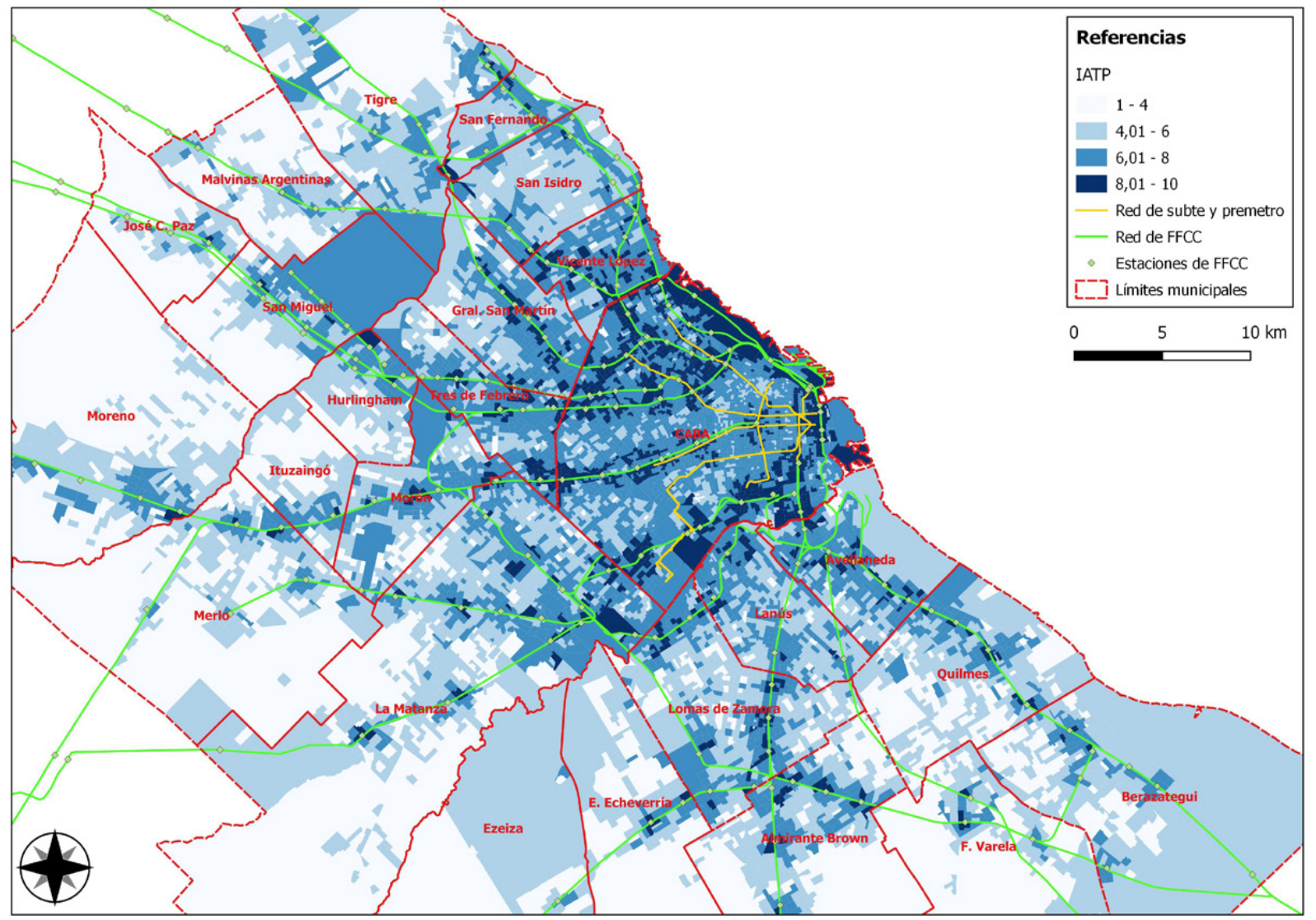

Berazategui, Ezeiza, San Miguel, La Matanza, Malvinas Argentinas y José C. Paz el tiempo promedio de viaje al trabajo oscila entre los 45 minutos y una hora. Por último, en los partidos de Florencio Varela, Merlo y Moreno los tiempos de viaje promedio son mayores a una hora.

En los sectores de los partidos del segundo y tercer cordón donde el tiempo promedio de viaje es menor a 45 minutos, lo más factible es que una mayor proporción de los viajes originados en esos barrios y localidades tengan como destino centros de empleo dentro del mismo partido o a lo sumo en partidos aledaños, y no el centro de CABA.

\section{Accesibilidad diferencial al transporte}

La hipótesis central de este estudio sostiene que el grado de accesibilidad al transporte público por parte de los hogares influye significativamente en los niveles de empleo. El desempleo no solo sería resultado de las capacidades técnicas de los individuos y de procesos económicos estructurales, sino de factores espaciales (localización) y temporales (tiempo de viaje al trabajo) que influyen en el nivel de acceso al mercado de trabajo por parte de los demandantes de empleo. La Figura 4 ilustra la distribución del IATP, construido con el fin de analizar esta dimensión en el territorio a nivel radio censal.

Se observa que la accesibilidad al transporte público por parte de los hogares de acuerdo al IATP tiende a ser mayor en los sectores de la CABA donde hay estaciones de subterráneo y/o ferrocarril próximas, en la primera corona del conurbano bonaerense y en las trazas de las líneas interurbanas de ferrocarril. En el IATP inciden a su vez la cercanía de los hogares a recorridos de colectivos y la suma de la longitud (en metros) de los recorridos de los colectivos del RC / densidad demográfica del RC.
Figura 4. Índice de Accesibilidad al Transporte Público. Fuente: Elaboración en base a relevamiento y cálculos propios y datos del CNPHV 2010. 
Los hogares que habitan sectores del conurbano alejadas de las estaciones de ferrocarril y dependen exclusivamente del transporte automotor, con un IATP no mayor a 6, son la gran masa de hogares cuyo acceso a oportunidades de empleo podría verse reducido debido a la falta de conectividad. Los más desconectados son los partidos cuyo territorio prevalece en el tercer cordón del conurbano: La Matanza, Merlo, Moreno, José C. Paz. Malvinas Argentinas, y Florencio Varela, principalmente.

\section{Modelo de regresión lineal}

Se diseñó un modelo de regresión lineal utilizando la base de 12.685 radios censales, donde el porcentaje de personas desocupadas es la variable dependiente, y el IATP y el tiempo de viaje al trabajo promedio operan como variables independientes. Además de estas dos variables asociadas a la movilidad se incluyeron tres variables independientes operacionalizadas del CNPVH 2010: porcentaje de hogares con Necesidades Básicas Insatisfechas, porcentaje de personas que no asisten o asistieron al secundario, y porcentaje de hogares sin tenencia formal de la vivienda.

Cuadro 5. Variables incluidas en el modelo de regresión lineal. Fuente: Elaboración propia.

\begin{tabular}{|l|l|l|}
\hline Variable & Descripción & Origen \\
\hline z_des010* & Porcentaje de personas desocupadas en el RC & CNPHV 2010 \\
\hline z_nbi10 & Porcentaje de hogares con NBI en el RC & CNPHV 2010 \\
\hline z_nedu10 & Porcentaje de personas que no asisten o asistieron al secundario en el RC & CNPHV 2010 \\
\hline z_nten10 & Porcentaje de hogares sin tenencia formal de la vivienda en el RC & CNPHV 2010 \\
\hline IATP & $\begin{array}{l}\text { Índice de Accesibilidad al Transporte Público (del 1 al 10, siendo 10 la } \\
\text { máxima accesibilidad) }\end{array}$ & $\begin{array}{l}\text { Elaboración propia (cálculos } \\
\text { geográficos) }\end{array}$ \\
\hline tiempo_prom & Tiempo promedio de viaje al trabajo (minutos) & Elaboración propia (ENMODO) \\
\hline
\end{tabular}

*Variable dependiente

Cuadro 6. Resultados del modelo de regresión lineal. Fuente: Elaboración propia en base a resultados de Stata.

\begin{tabular}{|c|c|c|c|c|c|c|}
\hline \multirow{3}{*}{$\frac{\text { z_deso10 }}{\text { z_nbi10 }}$} & \multirow{3}{*}{$\begin{array}{l}\text { Coeficiente } \\
, 0074625\end{array}$} & \multirow{3}{*}{$\begin{array}{l}\text { Error estándar } \\
\text {,0027921 }\end{array}$} & \multirow{3}{*}{$\frac{\mathrm{t}}{2,67}$} & \multicolumn{3}{|c|}{ 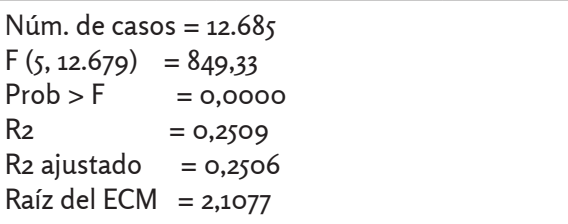 } \\
\hline & & & & \multirow{2}{*}{$\frac{P>|t|}{0,008}$} & \multicolumn{2}{|c|}{ [Intervalo de confianza 95\%] } \\
\hline & & & & & ,0019896 & ,0129354 \\
\hline z_nedu1o & ,2471131 & ,0119745 & 20,64 & 0,000 &, 2236413 &, 2705848 \\
\hline z_nten10 &, 0195245 & ,0026894 & 7,26 & 0,000 &, 0142528 &, 0247963 \\
\hline IATP &,- 2482968 & ,013246 & $-18,75$ & 0,000 &,- 2742609 &,- 2223327 \\
\hline tiempo_prom &, 0181243 & ,0017959 & 10,09 & 0,000 &, 014604 & ,0216446 \\
\hline constante & 4,548177 & 1499677 & 30,33 & 0,000 & 4,254218 & 4,842137 \\
\hline
\end{tabular}

En primer lugar, vale aclarar que este modelo es una aproximación inicial al estudio del tema. En principio, los resultados arrojados parecieran ser promisorios, pero para que el modelo sea efectivamente causal será necesario -en otros estudios- incluir otras dimensiones, como el precio del suelo, que podrían estar cofundando la relación (las zonas de menor precio del suelo suelen ser las de peor accesibilidad y de mayor desempleo). 

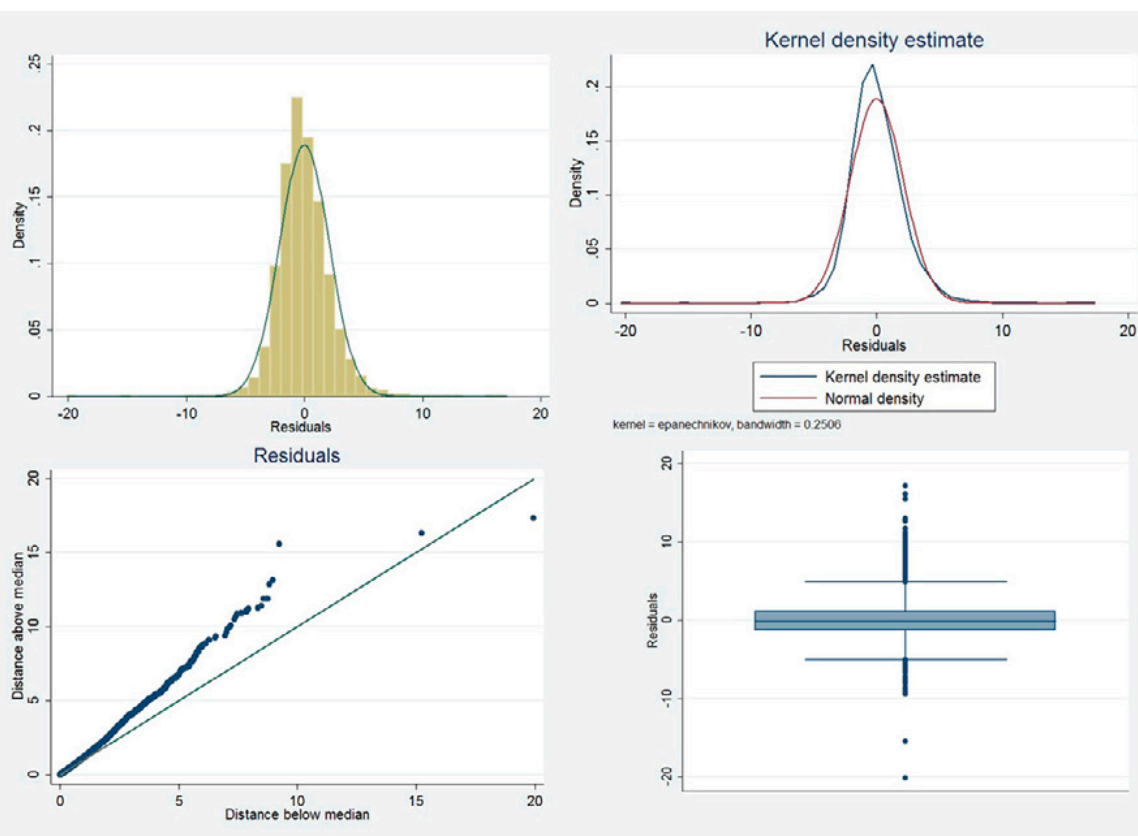

El modelo tiene un poder explicativo del $25 \%\left(\mathrm{R}^{2}=0,2509\right)$. Todas las variables son significativas al $1 \%$. Los resultados muestran que el aumento de un punto en el IATP implica un descenso de 0,248 puntos porcentuales en el porcentaje de personas desocupadas en el RC (z_deso10) o tasa de desocupación. Esto, en principio, avala la hipótesis central de este trabajo: a mayor accesibilidad al transporte público, menores niveles de desocupación en los hogares. El promedio de desocupación en la RGBA en 2010 era de $8,3 \%$. Esto implica que, en términos generales, el aumento de un punto en el IATP implica un descenso del 3\% sobre el valor promedio de la desocupación $(8,3$ $/ 0,248=0,03)$. Por ejemplo, una zona con un IATP equivalente a 5 tendría $12 \%$ más desocupación que una zona con 9 puntos de IATP.

Continuando con las otras variables del modelo, el tiempo promedio de viaje al trabajo (tiempo_prom) tiene una influencia positiva: el aumento de 1 minuto en el tiempo promedio de viaje al trabajo implica un aumento de 0,181 puntos porcentuales en la tasa de desocupación. A su vez, el nivel educativo (z_nedu10) también tiene influencia positiva: el aumento de un punto porcentual en el porcentaje de personas que no asisten o asistieron al secundario en el radio censal implica un aumento de 0,247 puntos porcentuales en la tasa de desocupación en ese radio censal. Por su parte, el régimen de tenencia de la vivienda tiene una influencia menor, aunque significativa: el aumento de un punto porcentual en el porcentaje de hogares sin tenencia formal de la vivienda en el radio censal implica un aumento de 0,195 puntos porcentuales en la tasa de desocupación. Por último, el aumento de un punto porcentual del porcentaje de hogares con NBI en el radio censal implica un aumento de 0,007 puntos porcentuales en la tasa de desocupación. De acuerdo a este modelo, el nivel de pobreza estructural es el factor de menor influencia sobre la tasa de desocupación, siendo la accesibilidad al transporte público y el nivel educativo los factores que ejercen el mayor peso.

En este sentido, la variable construida de accesibilidad al transporte público es significativa incluso controlando por otros factores: niveles de pobreza (NBI), nivel educativo, y régimen de tenencia de la vivienda. Sorprendentemente, el nivel de NBI es lo menos relevante en el modelo. Una explicación posible es que esta dimensión ya estaría capturada en las variables de educación y régimen de tenencia de la vivienda.
Figura 5. Residuos de la regresión. Fuente: Elaboración propia con Stata. 


\section{Reflexiones finales}

Los resultados del modelo muestran que los niveles de accesibilidad a la infraestructura de transporte público por parte de los hogares de la RGBA tienen incidencia significativa en los niveles de empleo de dichos hogares. Se ha constatado empíricamente que a medida que mejora la accesibilidad al transporte público, disminuyen las tasas de desocupación. Un menor tiempo de viaje también se mostró asociado a menores niveles de desocupación. A su vez, se ha constatado que hay determinantes sociodemográficos de los hogares - principalmente el nivel educativo - que también tienen incidencia significativa sobre las tasas de desocupación.

En vista de los resultados obtenidos, es insoslayable la importancia de la accesibilidad al transporte público por parte de los hogares residentes de la RGBA para garantizar el acceso - con un tiempo de viaje y costo razonables - a los centros de empleo.

La relación significativa encontrada entre los niveles de accesibilidad al transporte público y los niveles de desempleo en la región sugieren que una inversión en infraestructura de transporte en las zonas más relegadas - principalmente sectores de los partidos de Ezeiza, Merlo, La Matanza, Moreno, Almirante Brown, Florencio Varela, Malvinas Argentinas y José C. Paz - debe ser una prioridad para los gobiernos locales, provincial y nacional.

Partidos como La Matanza y Ezeiza han visto un notable crecimiento poblacional ( $41,5 \%$ y $37,8 \%$ de aumento entre 2001 y 2010 , respectivamente), viéndose superada ampliamente la oferta de transporte. En este sentido, resulta necesario un diagnóstico de la oferta de transporte público en los núcleos poblacionales que más han crecido en la región, con el objetivo de desarrollar un plan integral de movilidad y garantizar así el acceso de estos hogares al mercado de trabajo. Esto podría ser el eje de un próximo estudio una vez que estén disponibles los resultados del Censo Nacional programado para fines de 2020 .

Por último, la metodología desarrollada en este estudio puede ser replicada en otros aglomerados urbanos de la región con el fin de constatar que la accesibilidad al transporte público es un factor determinante en la igualdad de oportunidades de empleo y el mejoramiento de las condiciones de vida de la población. 


\section{Bibliografía}

» BLANCO, Jorge (2016) Urbanización y movilidad: contradicciones bajo el modelo automóvil - intensivo. Revista Transporte y Territorio n ${ }^{\circ}{ }_{15}$, Buenos Aires, pp. 96-113.

» CAF (2011) Desarrollo urbano y movilidad en América Latina. Panamá: Corporación Andina de Fomento.

»CNPHV (2010) CENSO NACIONAL DE POBLACIÓN, HOGARES Y VIVIENDAS 2010. INDEC, Ministerio de Economía.

»CNRT (2017) Informe Estadístico Anual 2016 - Red Ferroviaria de Pasajero del Área Metropolitana de Buenos Aires. Buenos Aires: Comisión Nacional de Regulación del Transporte. Gerencia Nacional de Regulación del Transporte. Área Estadística.

» CURRIE, Graham (2004) Gap analysis of public transport needs. Measuring spatial distribution of public transport needs and identifying gaps in the quality of public transport provision. Transportation Research Record vol. 1895, pp. 137-146.

» DGEyC (2013) DIRECCIÓN GENERAL DE ESTADÍSTICA Y CENSOS, MINISTERIO DE HACIENDA, GCBA. El mercado laboral porteño y su relación con el Conurbano: 4to. trimestre de 2012. Informe de resultados 578, Ciudad Autónoma de Buenos Aires.

» DGEyC (2017) DIRECCIÓN GENERAL DE ESTADÍSTICA Y CENSOS, MINISTERIO DE HACIENDA, GCBA. Anuario Estadístico 2016. Ciudad Autónoma de Buenos Aires.

»DGEyC (2017b)DIRECCIÓNGENERALDEESTADÍSTICA YCENSOS, MINISTERIO DE HACIENDA, GCBA. Indicadores laborales - ETOI. Caracterización de la PEA y Análisis Específico. Cuarto trimestre de 2016. Informe de resultados 1125. Ciudad Autónoma de Buenos Aires.

»ENMODO (2012) ENCUESTA DE MOVILIDAD DOMICILIARIA. Resultados de la Encuesta Origen Destino 2009-2010. Secretaría de Transporte de la Nación.

» ENNIS, Huberto y PORTO, Alberto (1997) Decisiones de localización y lugar de trabajo. Anales de la Reunión de la Asociación de Economía Política, Bahía Blanca, Argentina.

» GABRIEL, Stuart y ROSENTHAL, Stuart (1996) Commutes, Neighborhood Effects, and Earnings: An Analysis of Racial Discrimination and Compensating Differentials. Journal of Urban Economics, Julio, 40(1), pp. 61-83.

» GUTIÉRREZ, Andrea y REARTE, Julio (2006) Segregación y accesibilidad a servicios públicos de transporte en la Ciudad de Buenos Aires. Panorama Nacional da Pesquisa em Transportes. Rio de Janeiro, Brasil: ANPET, pp. 829-840.

» KARLICH, Susana y GUTIÉRREZ, Andrea (2007) Más allá de la informalidad en el transporte de pasajeros: reflexiones sobre los servicios de charters en la RMBA. Revista Lavboratorio. Estudios sobre Cambio Estructural y Desigualdad Social (CEyDS/UBA). Año 8, Núm. 20. Buenos Aires.

" LATTE, Alfredo (1980) El impacto del crecimiento demográfico en los mercados de trabajo rurales y urbano. CENEP. Cuaderno del CENEP N ${ }^{12 .}$ Buenos Aires, 1980.

» LUCAS, Karen, MATTIOLI, Giulio, VERLINGHIERI, Ersilia y GUZMÁN, Alvaro (2016) Transport poverty and its adverse social consequences. Proceedings of the Institute of Civil Engineers, pp. 353-365. 
" METROVÍAS (2017) Informe de gestión de subte y premetro 2016. Buenos Aires.

» MINOCHA Inshu, SRIRAJ P.S., METAXATOS, Paul, THAKURIAH, Piyushimita (2008) Analysis of Transit Quality of Service and Employment Accessibility for the Greater Chicago, Illinois, Region. Transportation Research Record: Journal of the Transportation Research Board, Num. 2042, Transportation Research Board of the National Academies, Washington, D.C., pp. 20-29.

"NEFFA, Julio (coord.) (2005) Actividad, empleo y desempleo: conceptos y definiciones. Miño y Dávila, CEIL-PIETTE CONICET. Buenos Aires.

" PESSINO, Carola y ANDRÉS, Luis (2000) La Dinámica Laboral en el Gran Buenos Aires y sus implicaciones para la Política Laboral y Social. Centro de Economía y Políticas Gubernamentales, Sociales y Laborales (CESyL), Universidad del CEMA.

»POK, Cynthia y LORENZETTI, Andrea (2007) El abordaje conceptualmetodológico de la informalidad. Revista Lavboratorio. Estudios sobre Cambio Estructural y Desigualdad Social (CEyDS/UBA). Año 8, Núm. 20. Buenos Aires.

"SMOLKA, Martim, BIDERMAN, Ciro (2011) Vivienda informal: una perspectiva de economista sobre el planeamiento urbano, Cambridge: Lincoln Institute of Land Policy.

"STANLEY, John, HENSHER, David, STANLEY, Janet, VELLA-BORDRICK, Dianne (2011) Mobility, social exclusion and well-being: Exploring the links. Transportation Research Part A, vol. 45, pp. 789-801.

\section{Fuentes de datos}

»Bases de microdatos del segundo trimestre de 2015 de la Encuesta Permanente de Hogares, INDEC https://www.indec.gob.ar/bases-de-datos.asp

»Buenos Aires Data (http://data.buenosaires.gob.ar)

»Capas geográficas con información censal 2001-2010 de la RGBA. Universidad Torcuato Di Tella.

»Capas geográficas con información sobre localidades bonaerenses. Instituto Geográfico Nacional. (http://www.ign.gob.ar/NuestasActividades/ sigign\#descarga)

»Encuesta de Movilidad Domiciliaria 2009-2010 (ENMODO). Bases de datos. Secretaría de Transporte de la Nación (http://uecmovilidad.gob.ar/encuestade-movilidad-domiciliaria-2009-2010-movilidad-en-el-area-metropolitana-debuenos-aires/)

»Open Street Maps (http://mapzen.com)

»Omnilíneas (http://omnilineas.com.ar)

\section{Alejandro Yomal / aleyomal@gmail.com}

Licenciado en Sociología por la Universidad de Buenos Aires y magister en Economía Urbana por la Universidad Torcuato di Tella. Se desempeña en el GCBA desde 2008: fue coordinador de comuna en la Dirección General de Estadística y Censos y analista en la Dirección General de Desarrollo Saludable. Actualmente es Gerente Operativo de Investigación y Participación en la Dirección General de Antropología Urbana de la Subsecretaría de Proyectos del Ministerio de Desarrollo Urbano y Transporte. 